\title{
A LINGUISTIC-VALUED WEIGHTED AGGREGATION OPERATOR TO MULTIPLE ATTRIBUTE GROUP DECISION MAKING WITH QUANTITATIVE AND QUALITATIVE INFORMATION
}

\author{
XIAOBING LI \\ Intelligent Control Development Center, Southwest Jiaotong University, \\ Chengdu 610031, P.R. China \\ DA RUAN \\ Belgian Nuclear Research Centre (SCK·CEN), \\ $2400 \mathrm{Mol} \&$ Ghent University, 9000 Gent, Belgium \\ JUN LIU \\ School of Computing and Mathematics, Faculty of Engineering, University of Ulster, \\ Jordanstown, Newtownabbey BT37 0QB, Northern Ireland, UK \\ YANG XU \\ Intelligent Control Development Center, Southwest Jiaotong University, \\ Chengdu 610031, P.R. China \\ Received: 14-04-2008 \\ Revised: $\quad 30-06-2008$
}

\begin{abstract}
In selecting an optional alternative in an environment of multiple attribute group decision making, different attributes of the alternative are often considered as with quantitative and qualitative information. Consequently, decision making problems may include preference information in different formats. In this paper, a lattice-based linguistic-valued weighted aggregation (LVWA) operator is proposed for multiple attribute group decision making with non-totally ordered linguistic-valued information. Then some transformation functions for unifying different formats of preference information are reviewed and summarized. Finally, an example is illustrated how to use the LVWA operator and transformation functions for multiple attribute group decision making.
\end{abstract}

Keywords: Transformation functions, multiple attribute group decision making, linguistic-valued weighted aggregation (LVWA) operator, linguistic-valued lattice implication algebra, lattice theory

\section{Introduction}

Multiple attribute group decision making (MAGDM) addresses the problems of choosing an optimal choice that has the highest degree of satisfaction by multiple experts' assessments from a set of alternatives that are characterized in terms of their attributes. Generally, multiple attribute group decision making problems follow a common scheme composed by the three phases:

(1) Evaluation phase: Experts are asked to give preference values to each attribute of each alternative.
(2) Aggregation phase: It combines individual preference values to obtain a collective preference value for each alternative.

(3) Exploitation phase: It orders the collective preference values to obtain the best alternatives.

In the first phase, experts are asked to provide their preferences on each attribute of each alternative. Usually, the information is expressed by means of numerical values such as exact values, interval values, fuzzy numbers, etc. However, in real world, human beings are constantly making decisions under a linguistic environment. For example, when evaluating the "comfort" or "design" of a car, linguistic labels like "good ", "fair", "poor" are usually used; evaluating a 
the speed of a car, linguistic labels like "very fast", "fast", "slow" can be used, and evaluating students' performances in their courses, linguistic labels like "bad", "medium", "good" can be used. As a result, it is necessary to consider aggregations of linguistic information.

To date, several methods have been proposed for dealing with linguistic information, for instance:

(1) The extension principle based method for operations on fuzzy numbers that support the semantics of the linguistic labels ${ }^{14,15}$.

(2) The symbolic method for computations on the indexes of the linguistic terms ${ }^{16}$; both the methods (1) and (2) process the results in the initial expression domains, which produce the consequent loss of information and hence the lack of precision ${ }^{17}$.

(3) A fuzzy linguistic model based method for the linguistic information with a pair of values called 2tuple, composed by a linguistic term and a number ${ }^{17-21}$. Along with the model, this method deals with the 2tuple without loss of information.

(4) The direct computing with words method ${ }^{1-3}$.

In this paper, we follow the 4 th method to aggregate linguistic-valued information for group decision making. At present, a number of researches have focused on group decision making with linguistic preference. Herrera et al. developed a consensus model for group decision making under linguistic assessments $^{7}$ and combined the linguistic ordered weighted averaging (LOWA) operator with linguistic preference relations and the concept of dominance and non-dominance to show its use in the field of group decision making based on the LOWA operator ${ }^{8}$. Later, Herrera et al. presented a consensus model in complete linguistic framework for group decision making guided by consistency and consensus measures ${ }^{9}$. Z.S. Xu proposed an uncertain linguistic ordered weighted averaging (ULOWA) aggregation operator and uncertain linguistic hybrid aggregation (ULHA) operator, and developed an approach to multiple attribute group decision making with uncertain linguistic information based on the ULOWA and ULHA operators ${ }^{10}$.

Although there are many aggregation operators to aggregate linguistic information, they can only aggregate linearly ordered linguistic information. Note that there exist incomparable linguistic terms, such as slightly false and very true. So it is necessary to find an algebra for modeling the ordering relation of the natural language terms.

Lattice theory is a well-developed branch of an abstract algebra for modeling the ordering relation in the real world. Lattice-valued algebra for modeling linguistic values would be a possible choice. To establish theories and methods to simultaneously deal with fuzziness and incomparability of processed object itself and uncertainty in the course of information processing, $\mathrm{Xu}$ combined a lattice with implication algebra and established the lattice implication algebra $^{24}$, which provides a necessary foundation for the processing of incomparable information. In addition, there are some research works on incomparable information processing. An evaluation method with incomparable information is presented in Ref. 13. Lattice-valued linguistic-based decision making method is discussed in Ref. 22. A model for handling linguistic terms in the framework of latticevalued logic is presented in Ref. 4. In Ref. 30, the LVWA operator based on linguistic-valued lattice implication algebra is presented. In this paper, based on the LVWA operator, an approach to solve multiple attribute group decision making with incomparable linguistic-valued information is established. In Ref. 11, a new method for sensory evaluation of industrial products with uncertain information is presented. In this approach, sensory data provided by different evaluators are transformed into measures of consistency on fuzzy satisfaction degrees. Based on these measures of consistency, the aggregated information for all evaluators and all attributes and measure the dissimilarity between evaluators and between used evaluations attributes is obtained. The effectiveness of this method has been validated in the fabric hand evaluation for a number of samples of knitted cotton.

On the other hand, in multiple attribute group decision making, different types of attributes, either quantitative or qualitative, need to be considered. Therefore, the decision making problems may include many different types of preference information such as number, interval and linguistic values. In order to deal with these preference information in different formats, some researches have been done ${ }^{26-29}$. This paper also aims at developing a new method for unifying 
preference information in different formats into the format for linguistic-valued information.

The paper is organized as follows: Section 2 briefly gives basic definitions of lattice implication algebra and linguistic-valued lattice implication algebra. Section 3 introduces the LVWA operator and discusses its properties. Section 4 studies transformation functions for unifying preference information in different formats. Section 5 proposes an approach for multiple attribute group decision making based on the LVWA operator with a linguistic-valued lattice implication algebra preference set. Section 6 illustrates how to use the proposed approach. The paper is concluded in Section 7.

\section{Preliminaries}

In this section, we recall some basic concepts about lattice implication algebra ${ }^{24}$ and linguistic truth-valued lattice implication algebra ${ }^{25}$. For some details of lattice implication algebra, we refer to Ref. 6 .

\subsection{Lattice Implication Algebra}

Definition 2.1 Let $(L, \vee, \wedge, ')$ be a bounded lattice with an order-reversing involution " ' " and the universal bounds $O, I, \rightarrow: L \times L \rightarrow L$ be a mapping. $\left(L, \vee, \wedge,{ }^{\prime}, \rightarrow, O, I\right)$ is called a lattice implication algebra if the following axioms hold for all $x, y, z \in L$ :

$\left(\mathrm{I}_{1}\right) x \rightarrow(y \rightarrow z)=y \rightarrow(x \rightarrow z)$;

$\left(\mathrm{I}_{2}\right) x \rightarrow x=I$;

$\left(\mathrm{I}_{3}\right) x \rightarrow y=y^{\prime} \rightarrow x^{\prime}$;

$\left(\mathrm{I}_{4}\right) x \rightarrow y=y \rightarrow x=I$ implies $x=y$;

$\left(\mathrm{I}_{5}\right)(x \rightarrow y) \rightarrow y=(y \rightarrow x) \rightarrow x$.

$\left(\mathrm{I}_{6}\right)(x \vee y) \rightarrow z=(x \rightarrow z) \wedge(y \rightarrow z)$;

$\left(\mathrm{I}_{7}\right)(x \wedge y) \rightarrow z=(x \rightarrow z) \vee(y \rightarrow z)$;

Theorem 2.1 Let $L$ be a lattice implication algebra. Then for any $x, y, z \in L$ :

(1) If $y \leq z$, then $x \rightarrow y \leq x \rightarrow z$;

(2) If $x \leq y$, then $x \rightarrow z \geq y \rightarrow z$;

(3) $O \rightarrow x=I$;

(4) $I \rightarrow x=x$;

(5) $x \leq y$ if and only if $x \rightarrow y=I$;

(6) $x \rightarrow y \geq x^{\prime} \vee y$.

Example 2.1 (Boolean algebra) Let $\left(L, \vee, \wedge,,^{\prime}\right)$ be a Boolean lattice. For any $x, y \in L$, define

$$
x \rightarrow y=x^{\prime} \vee y \text {. }
$$

Then $\left(L, \vee, \wedge,{ }^{\prime}, \rightarrow\right)$ is a lattice implication algebra.

Example 2.2 (Lukasiewicz implication algebra on finite chains) Consider a set $L=\left\{a_{i} \mid i=1,2, \cdots, n\right\}$. For any $1 \leq j, k \leq n$, define

$$
\begin{aligned}
& a_{j} \vee a_{k}=a_{\max \{j, k\}}, \\
& a_{j} \wedge a_{k}=a_{\min \{j, k\}}, \\
& \left(a_{j}\right)^{\prime}=a_{n-j+1}, \\
& a_{j} \rightarrow a_{k}=a_{\min \{n-j+k, n\}} .
\end{aligned}
$$

Then $\left(L, \vee, \wedge,{ }^{\prime}, \rightarrow\right)$ is a lattice implication algebra.

In the following sections, the lattice implication algebra $\left(L, \vee, \wedge,{ }^{\prime}, \rightarrow\right)$ is denoted by $L$ simply unless emphasized.

\subsection{Linguistic-valued lattice implication algebra}

Definition 2.2 Let $\left(L_{i}, \vee_{i}, \wedge_{i}, \rightarrow_{i}, I_{i}, O_{i}\right) \quad(i=1,2, \mathrm{~L}, n)$ be a family of lattice implication algebras. Then

$$
L=\prod_{i=1}^{n} L_{i}=\left\{\left(a_{1}, a_{2}, \mathrm{~L}, a_{n}\right) \mid a_{i} \in L_{i}\right\}
$$

is called a direct product of $n$ lattice implication algebras.

Theorem 2.2 Let $L_{i} \quad(i=1,2, \mathrm{~L}, n)$ be a lattice implication algebra. If the operators $\vee, \wedge, ', \rightarrow$ on $L=\prod_{i=1}^{n} L_{i}$ are defined as follows respectively: for any

$$
\begin{gathered}
\left(a_{1}, a_{2}, \mathrm{~L}, a_{n}\right),\left(b_{1}, b_{2}, \mathrm{~L}, b_{n}\right) \in \prod_{i=1}^{n} L_{i}, \\
\left(a_{1}, a_{2}, \mathrm{~L}, a_{n}\right) \vee\left(b_{1}, b_{2}, \mathrm{~L}, b_{n}\right) \\
=\left(a_{1} \vee b_{1}, a_{2} \vee b_{2}, \mathrm{~L}, a_{n} \vee b_{n}\right), \\
\left(a_{1}, a_{2}, \mathrm{~L}, a_{n}\right) \wedge\left(b_{1}, b_{2}, \mathrm{~L}, b_{n}\right) \\
=\left(a_{1} \wedge b_{1}, a_{2} \wedge b_{2}, \mathrm{~L}, a_{n} \wedge b_{n}\right), \\
\left(a_{1}, a_{2}, \mathrm{~L}, a_{n}\right) \rightarrow\left(b_{1}, b_{2}, \mathrm{~L}, b_{n}\right) \\
=\left(a_{1} \rightarrow b_{1}, a_{2} \rightarrow b_{2}, \mathrm{~L}, a_{n} \rightarrow b_{n}\right), \\
\left(a_{1}, a_{2}, \mathrm{~L}, a_{n}\right)^{\prime}=\left(a_{1}^{\prime}, a_{2}^{\prime}, \mathrm{L}, a_{n}^{\prime}\right),
\end{gathered}
$$

then $\left(L, \vee, \wedge,{ }^{\prime}, \rightarrow\right)$ is also a lattice implication algebra.

Remark 2.1 Let $L=L_{1} \times L_{2}$ where $L_{i} \quad(i=1,2)$ be a finite-chain-type lattice implication algebra. Then $L$ is a lattice implication algebra.

Definition 2.3 Let $\mathrm{ML}=\left\{b_{1}, b_{2}\right\}$ be a linguistic term set, where $b_{1}$ be an antonym of $b_{2}$, and $b_{1} \leq b_{2}$ in term of their meanings in natural language, such as "poor" and "good", "false" and "true" etc. Define the same 
operators on ML as the ones in Example 2.1. Then ML is a lattice implication algebra, called a meta-linguistic lattice implication algebra.

Example 2.3 Let $M L=\{$ good, poor $\}$. The operators on ML are defined as the same in Example 2.1. Then ML is a meta-linguistic lattice implication algebra.

Definition 2.4 Let $\mathrm{MW}=\left\{a_{i} \mid i=1,2, \mathrm{~L}, n\right\}$, and $a_{i}$ $(i=1,2, \mathrm{~L}, n)$ be linguistic modifiers, used to modify the meta-linguistic terms. The set MW is ordered in the sense that $a_{i} \leq a_{j}$ if and only if $i \leq j$. The operators $\vee, \wedge, ', \rightarrow$ on MW are defined as the same in Example 2.2, then $\left(\mathrm{MW}, \vee, \wedge,{ }^{\prime}, \rightarrow, a_{1}, a_{n}\right)$ is a lattice implication algebra, called a lattice implication algebra with modifiers.

Example 2.4 Let $\mathrm{MW}=$ \{absolutely (Abbr. to $\mathrm{Ab}$ ), highly (Abbr. to Hi), very (Abbr. to Ve), quite (Abbr. to $\mathrm{Qu}$ ), exactly (Abbr. to $\mathrm{Ex}$ ), almost (Abbr. to $\mathrm{Al}$ ), rather (Abbr. to Ra), somewhat (Abbr. to So), slightly (Abbr. to S1) $\}$ be a set of linguistic modifiers. Then the chain $\mathrm{Ab} \geq \mathrm{Hi} \geq \mathrm{Ve} \geq \mathrm{Qu} \geq \mathrm{Ex} \geq \mathrm{Al} \geq \mathrm{Ra} \geq \mathrm{So} \geq \mathrm{Sl}$ is $\mathrm{a}$ linguistic-modifier lattice implication algebra with operations defined as in Example 2.2.

Definition 2.5 Let $\mathrm{MW}=\left\{a_{1}, a_{2}, \mathrm{~L}, a_{n}\right\}$ be a lattice implication algebra with modifiers, $\mathrm{ML}=\left\{b_{1}, b_{2}\right\}$ be a meta lattice implication algebra, denote $L_{n \times 2}=\left\{\left(a_{1}, b_{1}\right), \mathrm{L},\left(a_{n}, b_{1}\right),\left(a_{1}, b_{2}\right), \mathrm{L},\left(a_{n}, b_{2}\right)\right\}$, which Hasse diagram is shown as Fig.1.

The operations on $L_{n \times 2}$ is defined as follows:

$$
\begin{gathered}
\left(a_{i}, b_{j}\right)^{\prime}=\left(a_{i}^{\prime}, b_{j}^{\prime}\right), \\
\left(a_{i}, b_{j}\right) \rightarrow\left(a_{k}, b_{l}\right)=\left(a_{i} \rightarrow a_{k}, b_{j} \rightarrow b_{l}\right) .
\end{gathered}
$$

Define a mapping $f$ :

$$
\begin{array}{r}
f: \mathrm{MW} \times \mathrm{ML} \rightarrow L_{n \times 2} \\
f\left(\left(a_{i}, b_{j}\right)\right)=\left\{\begin{array}{ll}
\left(a_{i}^{\prime}, b_{j}\right), & b_{j}=b_{1} \\
\left(a_{i}, b_{j}\right), & b_{j}=b_{2}
\end{array} .\right.
\end{array}
$$

Then $f$ is a isomorphic mapping.

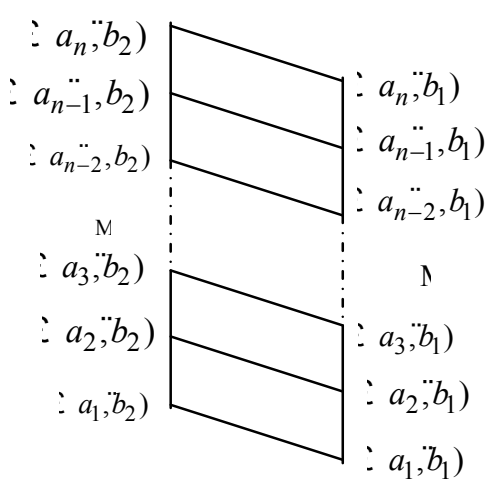

Figure 1. Hasse Diagram of $L_{n \times 2}$.

Define the operations on MW $\times$ ML as follows: $\left(a_{i}, b_{j}\right) \vee\left(a_{k}, b_{l}\right)=f^{-1}\left(f\left(a_{i}, b_{j}\right) \vee f\left(a_{k}, b_{l}\right)\right)$, $\left(a_{i}, b_{j}\right) \wedge\left(a_{k}, b_{l}\right)=f^{-1}\left(f\left(a_{i}, b_{j}\right) \wedge f\left(a_{k}, b_{l}\right)\right)$, $\left(a_{i}, b_{j}\right) \rightarrow\left(a_{k}, b_{l}\right)=f^{-1}\left(f\left(a_{i}, b_{j}\right) \rightarrow f\left(a_{k}, b_{l}\right)\right)$, $\left(a_{i}, b_{j}\right)^{\prime}=f^{-1}\left(\left(f\left(a_{i}, b_{j}\right)\right)^{\prime}\right)$.

Then $\mathrm{MW} \times \mathrm{ML}$ is called linguistic-valued lattice implication algebra, which Hasse diagram is shown in Fig. 2 .

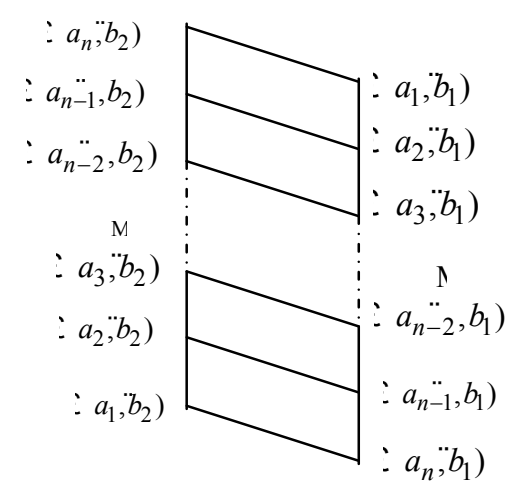

Figure 2. Hasse Diagram of MW $\times$ ML.

Example 2.5 Let $\mathrm{MW}=\{$ absolutely, highly, very, quite, exactly, almost, rather, somewhat, slightly $\}$ be a set of linguistic modifiers, and $\mathrm{ML}=\{$ good, poor $\}$. So $\mathrm{MW} \times \mathrm{ML}=\{$ absolutely good, highly good, very good, quite good, exactly good, almost good, rather good, somewhat good, slightly good, absolutely poor, highly poor, very poor, quite poor, exactly poor, almost poor, rather poor, somewhat poor, slightly poor $\}$. Then 
$(\mathrm{MW} \times \mathrm{ML}, \vee, \wedge, ', \rightarrow, O$ (i.e., slightly poor), $I$ (i.e., absolutely good) is a linguistic-valued lattice implication algebra. In the following section, we will use this linguistic-valued lattice implication algebra as a linguistic assessment set to represent the preference, or the important weight, denoted shortly as $S$.

\section{A linguistic-valued aggregation operator for multiple attribute group decision making}

Yager introduced an ordered weighted averaging (OWA) operator defined as follows ${ }^{5}$.

Definition 3.1 An OWA operator of dimension $n$ is a mapping OWA: $R^{n} \rightarrow R$ that has associated an $\mathrm{n}$ vector $\quad w=\left(w_{1}, w_{2}, \mathrm{~L}, w_{n}\right) \quad$ such that $w_{j} \in[0,1], j=1,2, \mathrm{~L}, n$, and $\sum_{j=1}^{n} w_{j}=1$.

Furthermore,

$$
\operatorname{OWA}_{w}\left(a_{1}, a_{2}, \mathrm{~L}, a_{n}\right)=\sum_{j=1}^{n} w_{j} b_{j},
$$

where $b_{j}$ is the $j$ th largest of the $a_{j}$.

However, the OWA operator can only be used in the situations where the input arguments are the exact numerical values. In the real world, human beings are constantly making decisions under a linguistic environment. Hence, it is necessary to investigate linguistic-valued information aggregation.

Remark 3.1: There have been some existing works on linguistic-valued information aggregation including Yager's work, such as Refs. 1, 2, 4, 5, but they are all based on the totally ordered linguistic term set.

In the following, we shall investigate a linguisticvalued weighted aggregation operator, which can be used in situations where the aggregated arguments are given in the form of linguistic values which may be incomparable.

Definition 3.2 A mapping LVWA: $S^{n} \rightarrow S$ is called a linguistic-valued weighted aggregation (LVWA) operator, if

$$
\operatorname{LVWA}_{w}\left(a_{1}, a_{2}, \mathrm{~L}, a_{n}\right)=\stackrel{n}{\wedge_{j=1}^{n}}\left(w_{j} \rightarrow a_{j}\right)
$$

where $S$ is an evaluation set which is a linguisticvalued lattice implication algebra and includes both comparable and incomparable linguistic terms commonly used in natural language, where $w=\left(w_{1}, w_{2}, \mathrm{~L}, w_{n}\right)$ is a weight vector and $w_{j}$ is the weight of linguistic-valued $a_{j}$ with $w_{j} \in S, a_{j} \in S$ and $j=1,2, \mathrm{~L}, n$.

Remark 3.2: Yager's aggregation method in Ref. 23 is a special case of the proposed method which is limited to the totally ordered linguistic term set.

The LVWA operator has the following properties:

Theorem 3.1 (Monotonicity) Let $A=\left(a_{1}, a_{2}, \mathrm{~L}, a_{n}\right)$ and $C=\left(c_{1}, c_{2}, \mathrm{~L}, c_{n}\right)$ be argument vectors. If for each $j \quad(j=1,2, \mathrm{~L}, n) \quad, \quad a_{j} \geq c_{j}$, then LVWA ${ }_{w}(A) \geq \operatorname{LVWA}_{w}(C)$.

Proof. Since

$$
\begin{aligned}
& \operatorname{LVWA}_{w}(A)=\underset{j=1}{\wedge}\left(w_{j} \rightarrow a_{j}\right), \text { and } \\
& \operatorname{LVWA}_{w}(C)=\underset{j=1}{n}\left(w_{j} \rightarrow c_{j}\right),
\end{aligned}
$$

the result follows directly from the property $a_{j} \geq c_{j}$.

Theorem 3.2 (Commutativity) Let $A=\left(a_{1}, a_{2}, \mathrm{~L}, a_{n}\right)$ be an ordered argument vector, $\bar{A}=\left(\bar{a}_{1}, \bar{a}_{2}, \mathrm{~L}, \bar{a}_{n}\right)$ is any permutation of the elements in $A$, then $\operatorname{LVOWA}_{w}(A)=\operatorname{LVOWA}_{w}(\bar{A})$.

Proof. Suppose that $w=\left(w_{1}, w_{2}, \mathrm{~L}, w_{n}\right)$ is the weighting vector of linguistic-valued $a_{j}(j=1,2, \mathrm{~L}, n)$. Then

$$
\begin{aligned}
& \operatorname{LVWA}_{w}\left(a_{1}, a_{2}, \mathrm{~L}, a_{n}\right)={\underset{j=1}{n}\left(w_{j} \rightarrow a_{j}\right) ;}_{\mathrm{LVWA}_{w}\left(\bar{a}_{1}, \bar{a}_{2}, \mathrm{~L}, \bar{a}_{n}\right)=\wedge_{j=1}^{n}\left(w_{j} \rightarrow \bar{a}_{j}\right) .}
\end{aligned}
$$

Hence,

$$
\operatorname{LVOWA}_{w}(A)=\operatorname{LVOWA}_{w}(\bar{A}) .
$$

Theorem 3.3 (Idempotence) If $\underset{j=1}{\vee} w_{j}=I$, and $a_{j}=a$ $(j=1,2, \mathrm{~L}, n)$, then

$$
\operatorname{LVWA}_{w}\left(a_{1}, a_{2}, \mathrm{~L}, a_{n}\right)=a .
$$

Proof. Since $a_{j}=a$, it follows that

$$
\begin{aligned}
& \operatorname{LVWA}_{w}\left(a_{1}, a_{2}, \mathrm{~L}, a_{n}\right)=\underset{j=1}{n}\left(w_{j} \rightarrow a_{j}\right) \\
& =\wedge_{j=1}^{n}\left(w_{j} \rightarrow a\right)=\left(\underset{j=1}{\vee} w_{j}\right) \rightarrow a=I \rightarrow a=a .
\end{aligned}
$$


Theorem 3.4 Let $w=(I, I, \mathrm{~L}, I)$. Then

$$
\operatorname{LVWA}_{w}\left(a_{1}, a_{2}, \mathrm{~L}, a_{n}\right)=\inf _{j}\left[a_{j}\right] \text {. }
$$

\section{Transformation schemes for unifying different formats of preference information}

To obtain evaluation results of all the alternatives in multiple attribute group decision making, different formats of preference information need to be unified into a common format. The linguistic-valued preference information set $S$ is chosen as the common format. The unifying steps are given as follows:

Step 1: normalization of quantitative preference information

Step 2: normalization of qualitative preference information

Step 3: transformation for numerical preference information to linguistic-valued preference information.

The concrete transformation methods will be given in the following subsections.

\subsection{Normalization of quantitative preference information}

Generally, there exist six kinds of attributes: profit, cost, fixation, interval, deviation, and deviating interval. In Refs. 27-29, the methods of normalizing the above six kinds of attributes are given and expressed as follows:

- Profit attribute

$$
\begin{aligned}
r_{i j} & =\frac{a_{i j}}{\max _{i}\left\{a_{i j}\right\}} \\
r_{i j} & =\frac{\min _{i}\left\{a_{i j}\right\}}{a_{i j}}
\end{aligned}
$$

- Cost attribute

- Fixation attribute $\quad r_{i j}=1-\frac{a_{i j}-\alpha_{j}}{\max _{i}\left\{a_{i j}-\alpha_{j}\right\}}$

- Interval attribute

$$
r_{i j}=\left|a_{i j}-\beta_{j}\right|-\frac{\min _{i}\left|a_{i j}-\beta_{j}\right|}{\max _{i}\left|a_{i j}-\beta_{j}\right|-\min _{i}\left|a_{i j}-\beta_{j}\right|}
$$

- Deviation attribute

$$
r_{i j}=\left\{\begin{array}{cc}
1-\frac{\max \left(q_{1}^{j}-a_{i j}, a_{i j}-q_{2}^{j}\right)}{\max \left[q_{1}^{j}-\min _{i}\left(a_{i j}\right), \max _{i}\left(a_{i j}\right)-q_{2}^{j}\right]}, & a_{i j} \notin\left[q_{1}^{j}, q_{2}^{j}\right] \\
1, & a_{i j} \in\left[q_{1}^{j}, q_{2}^{j}\right]
\end{array}\right.
$$

- Deviating interval attribute

$$
r_{i j}=\left\{\begin{array}{cc}
\frac{\max \left(q_{1}^{j}-a_{i j}, a_{i j}-q_{2}^{j}\right)}{\max \left[q_{1}^{j}-\min _{i}\left(a_{i j}\right), \max _{i}\left(a_{i j}\right)-q_{2}^{j}\right]}, & a_{i j} \notin\left[q_{1}^{j}, q_{2}^{j}\right] \\
0, & a_{i j} \in\left[q_{1}^{j}, q_{2}^{j}\right]
\end{array}\right.
$$

where $a_{i j}$ denotes the original value of attribute $A_{j}$ for alternative $X_{i}, r_{i j}$ represents the normalized value of $a_{i j}, \alpha_{j}$ and $\beta_{j}$ are fixed values, $\left[q_{1}^{j}, q_{2}^{j}\right]$ is a interval. The larger the profit attribute value, the better the attribute, while the larger the cost attribute value, the worse the attribute. The fixation attribute means that the closer to a fixed value $\alpha_{j}$ attribute value, the better the attribute. Further, we can know that the closer to or included in an interval $\left[q_{1}^{j}, q_{2}^{j}\right]$ values, the better the attribute. The larger of the distance of deviation attribute values to a fixed value are, the better of the attribute is. Deviation interval attribute means that the larger the distance of deviation attribute values to a fixed interval, the better the attribute.

Remark 4.1 According to the above formula, the original values can be normalized within the interval $[0$, $1]$.

\subsection{Normalization of qualitative preference information}

The linguistic values are designed to express preference information of qualitative attributes by decision makers. In this paper, all linguistic values are selected from linguistic-valued lattice implication algebra $S$ defined in Example 2.5. Two kinds of attributes, profit and cost, are considered. The methods of qualitative attributes are given as follows:

- Profit attribute: in this case, as the preference information is expressed by linguistic values, we keep the original values as the normalized values.

- Cost attribute:

$$
r_{i j}=a_{i j}^{\prime}
$$

where $a_{i j}$ denotes the original value of attribute $A_{j}$ for alternative $X_{i}$, “', " is a negation operator, $r_{i j}$ represents the normalized value of $a_{i j}, a_{i j}$ and $r_{i j}$ are all linguistic values in $S$.

\subsection{Transformation function for numerical attribute values to linguistic-valued attribute values}

After the transformation of the original attribute values, normalized attribute values are expressed by number in 
interval $[0,1]$ or linguistic values in $S$. We need to unify these two kinds of preference information. Since the attribute values belonging to the interval $[0,1]$ are comparable, we select the subset $S^{*}=\left\{s_{0}, s_{1}, \mathrm{~L}, s_{n}\right\}$ of $S$ such that $S^{*}$ only contains comparable linguistic values and these linguistic values satisfy the following conditions:

- A negation operator “ ' ": $s_{i}^{\prime}=s_{j}$ such that $j=n-i(n+1$ is the cardinality)

- A min and a max operator in the linguistic term set: $s_{i} \leq s_{j} \Leftrightarrow i \leq j$

To aggregate the preference information, a transformation function for numerical attribute values to linguistic-valued attribute values is given as follows:

$$
\tau:[0,1] \rightarrow S^{*}, \tau(a)=S_{[n a]}
$$

where "[ ]" is the rounding operation, $n+1$ is the cardinality of $S^{*}$. Utilizing the transformation function, the numerical attribute values can be transferred into linguistic-valued attribute values.

\section{An approach based on the LVWA operator to multiple attribute decision making with linguistic-valued information}

Consider a multiple attribute group decision making problem with different formats of preference information. Assume that $S$ is an evaluation set that is a linguistic-valued lattice implication algebra and includes both comparable and incomparable natural linguistic terms used to indicate preference information. Let $X=\left\{x_{1}, x_{2}, \mathrm{~L}, x_{n}\right\}$ be a discrete set of alternatives, and $U=\left\{u_{1}, u_{2}, \mathrm{~L}, u_{m}\right\}$ be a set of attributes. Let $D=\left\{d_{1}, d_{2}, \mathrm{~L}, d_{l}\right\}$ be a set of decision makers, and $w=\left(w_{1}, w_{2}, \mathrm{~L}, w_{l}\right)$ be the weight vector of decision makers, where $w_{k} \in S, k=1,2, \mathrm{~L}, l$. Suppose that $\AA^{\left(b^{k}\right)}=\left(a_{i j}^{(k)}\right)_{m \times n}$ is the decision matrix, where $a_{i j}^{(k)}$ is a preference value, which takes the forms of number, interval, or linguistic value, given by the decision maker $d_{k} \in D$, for alternative $x_{j} \in X$ with respect to attributes $u_{i} \in U$. Group decision making problems are composed by the following four phases:

(1) Evaluation phase: The experts are asked to give the preference values to each attribute of each alternative.

(2) Transformation phase: All the preference values are expressed in a unique linguistic-valued domain.
(3) Aggregation phase: It combines the individual preferences to obtain a collective preference value for each alterative.

(4) Exploitation phase: It orders the collective preference values to obtain the best alternatives.

In the following an approach to multiple attribute group decision making with linguistic-valued information is given based on the LVWA operator.

Step 1: Experts give preference information $a_{i j}^{(k)}$, $i=1,2, \mathrm{~L}, m, j=1,2, \mathrm{~L}, n, k=1,2, \mathrm{~L}, l$.

Step 2: Utilize the decision information given in matrix $A^{0}\left(b^{k)}\right.$ and the methods of transformation in Section 4 to derive all the normalized linguistic values.

Step 3: Utilize the LVWA operator:

$$
a_{0}^{(k)}=\operatorname{LVWA}_{w}\left(a_{1 j}^{(k)}, a_{2 j}^{(k)}, \mathrm{L}, a_{m j}^{(k)}\right),
$$

$k=1,2, \mathrm{~L}, l, j=1,2, \mathrm{~L}, n$

to derive the individual overall preference value $a_{0}^{k k}$ of alternative $x_{j}$, where $w=\left(w_{1}, w_{2}, \mathrm{~L}, w_{m}\right)$ is a weight vector and $w_{j}$ is the weight of linguistic-valued $a_{j}$ with $w_{j} \in S, j=1,2, \mathrm{~L}, l$.

Step 4: Utilize the LVWA operator:

$\&_{\rho}=\mathrm{LVWA}_{w}\left(a_{j}^{(1)}, a_{j}^{(2)}, \mathrm{L}, a_{j}^{(l)}\right), j=1,2, \mathrm{~L}, n$

to derive the collective overall preference value $\mathscr{Q}_{0}$ of alternative $x_{j}$, where $w=\left(w_{1}, w_{2}, \mathrm{~L}, w_{l}\right)$ is the weight vector of decision makers, with $w_{j} \in S, j=1,2, \mathrm{~L}, n$.

Step 5: Rank all the alternatives $x_{j}$, and select the optimal one(s) in according to $\mathscr{\alpha}$. The optimal alternative is $x_{j} \in X$ that $\mathscr{Q}_{g}$ is maximal.

Step 6: End.

\section{An illustrative example}

To illustrate how the proposed method works, we will give a simple example ${ }^{23}$ to evaluate the set of cars $A=\left\{x_{1}=\right.$ Chevrolet, $x_{2}=$ Buick, $x_{3}=$ Toyota $\}$. Let $U=\left\{u_{1}, u_{2}, u_{3}, u_{4}\right\}$ where $u_{1}=$ comfort, $u_{2}=$ repair frequency, $u_{3}=$ cost, $u_{4}=$ maximum speed (whose vector weights be $\left.w=\left(\left(a_{7}, 1\right),\left(a_{9}, 0\right),\left(a_{8}, 1\right),\left(a_{6}, 1\right)\right)\right)$, and values of attributes $u_{1}$ and $u_{2}$ are linguistic values while values of attributes $u_{3}$ and $u_{4}$ are numerical values. Three kinds of cars (alternatives) $x_{j}(j=1,2,3)$ are to be evaluated using the term set 
$S=\left\{\left(a_{9}, 1\right)=\right.$ absolutely good, $\left(a_{8}, 1\right)=$ highly good, $\left(a_{7}, 1\right)=$ very good, $\left(a_{6}, 1\right)=$ quite good, $\left(a_{5}, 1\right)=$ exactly good, $\left(a_{4}, 1\right)=$ almost good, $\left(a_{3}, 1\right)=$ rather good, $\left(a_{2}, 1\right)=$ somewhat good, $\left(a_{1}, 1\right)=$ slightly good, $\left(a_{9}, 0\right)=$ slightly poor, $\left(a_{8}, 0\right)=$ somewhat poor, $\left(a_{7}, 0\right)=$ rather poor, $\left(a_{6}, 0\right)=$ almost poor, $\left(a_{5}, 0\right)=$ exactly poor,$\left(a_{4}, 0\right)=$ quite poor, $\left(a_{3}, 0\right)=$ very poor, $\left(a_{2}, 0\right)=$ highly poor, $\left(a_{1}, 0\right)=$ absolutely poor $\}$ by four decision makers $d_{k}(k=1,2,3,4)$ (whose weight vector $\left.\omega=\left(\left(a_{5}, 0\right),\left(a_{7}, 1\right),\left(a_{8}, 0\right),\left(a_{9}, 1\right)\right)\right)$ under these four attributes, as listed in Tables 1-4, respectively.

Step 1: Unify the attribute values into a linguistic values

Step 1.1: Utilize the transformation functions given in Section 4.1

$$
r_{i j}=\frac{a_{i j}}{\max _{i}\left\{a_{i j}\right\}} \text { and } r_{i j}=\frac{\min _{i}\left\{a_{i j}\right\}}{a_{i j}}
$$

to derive the normalized preference information of attribute $u_{3}$ and $u_{4}$ respectively.

Step 1.2: Utilize the transformation functions given in Section 4.2 to derive the normalized preference information of attribute $u_{1}$ and $u_{2}$ respectively.

Table 1. Preference information given by decision maker $d_{1}$

\begin{tabular}{llll}
\hline$u_{i}$ & $x_{1}$ & $x_{2}$ & $x_{3}$ \\
\hline$u_{1}$ & $\left(a_{9}, 1\right)$ & $\left(a_{7}, 1\right)$ & $\left(a_{6}, 1\right)$ \\
$u_{2}$ & $\left(a_{3}, 0\right)$ & $\left(a_{2}, 0\right)$ & $\left(a_{2}, 1\right)$ \\
$u_{3}$ & 150 & 120 & 200 \\
$u_{4}$ & 180 & 200 & 160 \\
\hline
\end{tabular}

Table 3. Preference information given by decision maker $d_{3}$

\begin{tabular}{llll}
\hline$u_{i}$ & $x_{1}$ & $x_{2}$ & $x_{3}$ \\
\hline$u_{1}$ & $\left(a_{8}, 1\right)$ & $\left(a_{6}, 0\right)$ & $\left(a_{9}, 1\right)$ \\
$u_{2}$ & $\left(a_{1}, 0\right)$ & $\left(a_{3}, 0\right)$ & $\left(a_{2}, 0\right)$ \\
$u_{3}$ & 150 & 120 & 200 \\
$u_{4}$ & 180 & 200 & 160 \\
\hline
\end{tabular}

Step 1.3: Utilize the transformation function given in Section 4.3 to unify all the preference information.

We chose the $S^{*}=\left\{s_{9}=\left(a_{9}, 1\right)=\right.$ absolutely good, $s_{8}=\left(a_{8}, 1\right)=$ highly good, $s_{7}=\left(a_{7}, 1\right)=$ very good, $s_{6}=\left(a_{6}, 1\right)=$ quite good, $s_{5}=\left(a_{5}, 1\right)=$ exactly good, $s_{4}=\left(a_{5}, 0\right)=$ exactly poor,$s_{3}=\left(a_{4}, 0\right)=$ quite poor, $s_{2}=\left(a_{3}, 0\right)=$ very poor, $s_{1}=\left(a_{2}, 0\right)=$ highly poor, $s_{0}=\left(a_{1}, 0\right)=$ absolutely poor $\}$. It is obvious that $S^{*}$ is a linear order.

After the above three steps, we can get Tables 5-8.

Step 2: Utilize the preference information given in Table 5 and the LVWA operator (Let $\left.w=\left(\left(a_{7}, 1\right),\left(a_{9}, 0\right),\left(a_{8}, 1\right),\left(a_{6}, 1\right)\right)\right)$

$a g^{(k)}=\mathrm{LVWA}_{w}\left(a_{p_{j}}^{(k)}, a_{q_{j}}^{(k)}, a_{3 j}^{(k)}, a_{q_{j}}^{(k)}\right)$,

$k=1,2,3,4, j=1,2,3$ to derive the individual overall preference value $8 g^{(k)}$ of the alternative $x_{j}$ :

$a_{p}^{(1)}=\operatorname{LVWA}_{w}\left(\left(a_{9}, 1\right),\left(a_{7}, 1\right),\left(a_{7}, 1\right),\left(a_{8}, 1\right)\right)$

$=\left(\left(a_{7}, 1\right) \rightarrow\left(a_{9}, 1\right)\right) \wedge\left(\left(\left(a_{9}, 0\right) \rightarrow\left(a_{7}, 1\right)\right) \wedge\left(\left(a_{8}, 1\right) \rightarrow\left(a_{7}, 1\right)\right)\right.$

$\wedge\left(\left(a_{6}, 1\right) \rightarrow\left(a_{8}, 1\right)\right)$

$=\left(a_{7}, 1\right)$

Similarly, we have

$a_{q}^{(1)}=\left(a_{8}, 1\right), a_{9}^{(1)}=\left(a_{6}, 1\right), a_{p}^{(2)}=\left(a_{6}, 1\right), a_{2}^{(2)}=\left(a_{8}, 0\right)$,

Table 2. Preference information given by decision maker $d_{2}$

\begin{tabular}{llll}
\hline$u_{i}$ & $x_{1}$ & $x_{2}$ & $x_{3}$ \\
\hline$u_{1}$ & $\left(a_{7}, 1\right)$ & $\left(a_{6}, 0\right)$ & $\left(a_{9}, 0\right)$ \\
$u_{2}$ & $\left(a_{4}, 0\right)$ & $\left(a_{1}, 1\right)$ & $\left(a_{1}, 1\right)$ \\
$u_{3}$ & 150 & 120 & 200 \\
$u_{4}$ & 180 & 200 & 160 \\
\hline
\end{tabular}

Table 4. Preference information given by decision maker $d_{4}$

\begin{tabular}{llll}
\hline$u_{i}$ & $x_{1}$ & $x_{2}$ & $x_{3}$ \\
\hline$u_{1}$ & $\left(a_{7}, 1\right)$ & $\left(a_{9}, 1\right)$ & $\left(a_{9}, 0\right)$ \\
$u_{2}$ & $\left(a_{2}, 1\right)$ & $\left(a_{3}, 0\right)$ & $\left(a_{2}, 0\right)$ \\
$u_{3}$ & 150 & 120 & 200 \\
$u_{4}$ & 180 & 200 & 160 \\
\hline
\end{tabular}


Table 5. Normalized reference information of Table 1

\begin{tabular}{llll}
\hline$u_{i}$ & $x_{1}$ & $x_{2}$ & $x_{3}$ \\
\hline$u_{1}$ & $\left(a_{9}, 1\right)$ & $\left(a_{7}, 1\right)$ & $\left(a_{6}, 1\right)$ \\
$u_{2}$ & $\left(a_{7}, 1\right)$ & $\left(a_{8}, 1\right)$ & $\left(a_{8}, 0\right)$ \\
$u_{3}$ & $\left(a_{7}, 1\right)$ & $\left(a_{9}, 1\right)$ & $\left(a_{5}, 1\right)$ \\
$u_{4}$ & $\left(a_{8}, 1\right)$ & $\left(a_{9}, 1\right)$ & $\left(a_{7}, 1\right)$ \\
\hline
\end{tabular}

Table 7. Normalized reference information of Table 3

\begin{tabular}{llll}
\hline$u_{i}$ & $x_{1}$ & $x_{2}$ & $x_{3}$ \\
\hline$u_{1}$ & $\left(a_{8}, 1\right)$ & $\left(a_{6}, 0\right)$ & $\left(a_{9}, 1\right)$ \\
$u_{2}$ & $\left(a_{9}, 1\right)$ & $\left(a_{7}, 1\right)$ & $\left(a_{8}, 1\right)$ \\
$u_{3}$ & $\left(a_{7}, 1\right)$ & $\left(a_{9}, 1\right)$ & $\left(a_{5}, 1\right)$ \\
$u_{4}$ & $\left(a_{8}, 1\right)$ & $\left(a_{9}, 1\right)$ & $\left(a_{7}, 1\right)$ \\
\hline
\end{tabular}

$a_{9}^{(2)}=\left(a_{6}, 0\right), a_{p}^{(3)}=\left(a_{8}, 1\right), a_{9}^{(3)}=\left(a_{7}, 0\right)$,

$a_{9}^{(3)}=\left(a_{6}, 1\right), a_{p}^{(4)}=\left(a_{8}, 1\right), a_{2}^{(4)}=\left(a_{7}, 1\right), a_{9}^{(4)}=\left(a_{6}, 0\right)$

Step 3: Utilize the weight vector of decision makers, $\omega=\left(\left(a_{5}, 0\right),\left(a_{7}, 1\right),\left(a_{8}, 0\right),\left(a_{9}, 1\right)\right) \quad, \quad$ and the LVWA operator:

$$
\ddot{d g}=\mathrm{LVWA}_{\omega}\left(a g_{g}^{(1)}, a g^{(2)}, a g_{g}^{(3)}, q_{g}^{(4)}\right)(j=1,2,3)
$$

to aggregate the individual overall preference values $a g^{k)}(k=1,2,3,4)$ and thus get the collective overall preference value $\mathscr{d} g$ of alternative $x_{j}$ :

$$
\begin{aligned}
& u_{p}=\operatorname{LVWA}_{\omega}\left(a_{p}^{(1)}, a_{p}^{(2)}, a_{p}^{(3)}, a_{p}^{(4)}\right) \\
& =\left(\left(a_{5}, 0\right) \rightarrow\left(a_{8}, 1\right)\right) \wedge\left(\left(a_{7}, 1\right) \rightarrow\left(a_{6}, 1\right)\right) \\
& \wedge\left(\left(a_{8}, 0\right) \rightarrow\left(a_{8}, 1\right)\right) \wedge\left(\left(a_{9}, 1\right) \rightarrow\left(a_{8}, 1\right)\right) \\
& =\left(a_{8}, 1\right)
\end{aligned}
$$

Similarly, we have

$$
q_{2}=\left(a_{7}, 0\right), d_{9}=\left(a_{6}, 0\right) \text {. }
$$

Step 4: Rank all the alternatives $x_{j}$, and select the optimal one(s) in according with $\& g$. The optimal alternative is $x_{j} \in X$ that $\mathscr{d g}$ is maximal. Thus the optimal one is $x_{1}$, that is Chevrolet.

\section{Conclusions}

In this paper, a linguistic-valued weighted aggregation operator was proposed, which can be used in the situations where the evaluation value set is a nontotally ordered linguistic term set, based on a linguistic-
Table 6. Normalized reference information of Table 2

\begin{tabular}{llll}
\hline$u_{i}$ & $x_{1}$ & $x_{2}$ & $x_{3}$ \\
\hline$u_{1}$ & $\left(a_{7}, 1\right)$ & $\left(a_{6}, 0\right)$ & $\left(a_{9}, 0\right)$ \\
$u_{2}$ & $\left(a_{6}, 1\right)$ & $\left(a_{9}, 0\right)$ & $\left(a_{9}, 0\right)$ \\
$u_{3}$ & $\left(a_{7}, 1\right)$ & $\left(a_{9}, 1\right)$ & $\left(a_{5}, 1\right)$ \\
$u_{4}$ & $\left(a_{8}, 1\right)$ & $\left(a_{9}, 1\right)$ & $\left(a_{7}, 1\right)$ \\
\hline
\end{tabular}

Table 8. Normalized reference information of Table 4

\begin{tabular}{llll}
\hline$u_{i}$ & $x_{1}$ & $x_{2}$ & $x_{3}$ \\
\hline$u_{1}$ & $\left(a_{7}, 1\right)$ & $\left(a_{9}, 1\right)$ & $\left(a_{9}, 0\right)$ \\
$u_{2}$ & $\left(a_{8}, 0\right)$ & $\left(a_{7}, 1\right)$ & $\left(a_{8}, 1\right)$ \\
$u_{3}$ & $\left(a_{7}, 1\right)$ & $\left(a_{9}, 1\right)$ & $\left(a_{5}, 1\right)$ \\
$u_{4}$ & $\left(a_{8}, 1\right)$ & $\left(a_{9}, 1\right)$ & $\left(a_{7}, 1\right)$ \\
\hline
\end{tabular}

valued lattice implication algebra. In order to deal with preference information in different formats, transformation methods were summarized. Finally, a method for a multiple attribute group decision making is developed based on the LVWA operator and transformation methods. Advantages of this approach are as follows:

(1) It does not require all linguistic terms to have a total order.

(2) It permits to compute with preference information in different formats.

\section{Acknowledgements}

We would like to express our thanks to the support of the China-Flanders Bilateral Project (Grant No. O11S1105), the National Natural Science Foundation of China (Grant No. 60474022) and the Specialized Research Foundation for the Doctoral Program of Higher Education of China (Grant No. 20060613007).

\section{References}

1. Z.S. $\mathrm{Xu}, \mathrm{A}$ method based on linguistic aggregation operators for group decision making with linguistic preference relations, Information Sciences, 166:19-30, 2004.

2. Z.S. Xu, EOWA and EOWG operators for aggregating linguistic labels based on linguistic preference relations, International Journal of Uncertainty, Fuzziness and Knowledge-Based Systems, 12:791-810, 2004. 
3. Z.S. Xu, Deviation measures of linguistic preference relations in group decision making, Omega, 33:249-254, 2005.

4. J. Ma, W.J. Li, Y. Xu and Z.M. Song, A model for handling linguistic terms in the framework of lattice-valued logic $L F(X), 2004$ IEEE International Conference On Systems, Man and Cybernetics, pp.1504-1509, 2004.

5. R.R. Yager, On ordered weighted averaging aggregation operations in multicriteria decision making, IEEE Transactions on Systems, Man, and Cybernetics, 18:183-190, 1988.

6. Y. Xu, D. Ruan, K.Y. Qin, and J. Liu, Latticevalued logic: an alternative approach to treat fuzziness and incomparability, Springer-Verlag, Heidelberg, 2003.

7. F. Herrera, L. Martinez, J.L. Verdegay, A model of consensus in group decision making under linguistic assessments, Fuzzy Sets and Systems, 78:73-87, 1996.

8. F. Herrera, L. Martinez, J.L Verdegay, Direct approach processes in group decision making using linguistic OWA operators, Fuzzy Sets and Systems, 79:175-190, 1996.

9. F. Herrera, L. Martinez, J.L. Verdegay, A rational consensus model in group decision making using linguistic assessments, Fuzzy Sets and System, 88:31-49, 1997.

10. Z.S. $\mathrm{Xu}$, Uncertain linguistic aggregation operators based approach to multiple attribute group decision making under uncertain linguistic environment, Information Science, 168:171-184, 2004.

11. Y. Xu, X. Y. Zeng, Ludovic Koehl An Intelligent Sensory Evaluation Method for Industrial Products Characterization, Int. J. of Information Technology \& Decision Making, 2007, 6(2): 349-370.

12. N. Bryson, A. Mobolurin, An action learning evaluation procedure for multiple criteria decision making problems, European Journal of Operational Research, 96:379-386, 1995.

13. D. Meng, H.D. Jia, Z.Q. Zhang, and Y. Xu, Linguistic truth-value lattice-valued logic system with important coefficient and its application to evaluation system, International Journal of Computer Science and Network Secutity, 6(6):1-6, 2006.

14. P.P. Bonissone, K.S. Decker, Selecting uncertainty calculi and granularity: an experiment in trading-off precision and complexity, in: L.H.
Kanal, J.F. Lemmer (Eds.), Uncertainty in Artificial Intelligence, North-Holland, Amsterdam, pp. 217-247, 1986.

15. R. Degani, G. Bortolan, The problem of linguistic approximation in clinical decision making, International Journal of Approximate Reasoning, 2:143-162, 1988.

16. M. Delgado, J.L. Verdegay, M.A. Vila, On aggregation operations of linguistic labels, International Journal of Intelligent Systems, 8: 351-370, 1993.

17. F. Herrera, L. Martı'nez, A 2-tuple fuzzy linguistic representation model for computing with words, IEEE Transactions on Fuzzy Systems, 8:746-752, 2000.

18. F. Herrera, L. Martı'nez, A model based on linguistic 2-tuples for dealing with multi-granular hierarchical linguistic contexts in multi-expert decision-making, IEEE transactions on systems, man, and cybernetics-part B, Cybernetics, 31: 227-234, 2001.

19. F. Herrera, L. Marti'nez, An approach for combining linguistic and numerical information based on 2-tuple fuzzy linguistic representation model in decision-making, International Journal of Uncertainty, Fuzziness, Knowledge-based Systems, 8:539-562, 2000.

20. M. Delgado, F. Herrera, E. Herrera-Viedma, M.J. Martin-Bautista, L. Martinez, M.A. Vila, A communication model based on the 2-tuple fuzzy linguistic representation for a distributed intelligent agent system on internet, Soft Computing, 6:320-328, 2002.

21. F. Herrera, L. Martinez, P.J. Sanchez, Managing non-homogeneous information in group decision making, European Journal of Operational Research, 166:115-132, 2005.

22. J. Liu, Y. Xu, D. Ruan, and L. Martinez, latticevalued linguistic-based decision making method, IEEE International Conference on Granular Computing(Grc 2005), (Beijing, China, July 2527, 2005), pp.199-202.

23. R.R. Yager, A new methodology for ordinal multiple objective decisions based on fuzzy sets, in : D. Dubios, H. Prade and R.R. Yager, eds., Fuzzy Sets for Intelligent System, San Maeto, CA: Morgan Kaufmann Publishers, pp.751-756, 1995.

24. Y. Xu. Lattice implication algebra, Journal of Southwest Jiaotong University, 89(1):20-27, 1993.(in Chinese) 
25. Y. Xu, S.W. Chen, and J. Ma, Linguistic truthvalued lattice implication algebra and its properties, IMACS Multi-conference on Computational Engineering in System Application, pp.1413-1418, 2006.

26. Luis Martinez, J Liu, D. Ruan, J. B. Yang, Dealing with heterogeneous information in engineering evaluation processes, Information Sciences, 177(7): 1533-1542.

27. Hwang $\mathrm{C} \mathrm{L}$ and Yoon K. Multiple attribute decision making- methods and applications. A State-of-the Art Survey. (Springer-Verlag, Berlin Herdelberg, 1981)
28. Z. T. Chen, Analysis of decision making. Scientific press, Beijing, 1987.

29. S. L. Liu, W. H. Qiu, Studies on the basic theories for MADM, System Engineering: Theory and Practice, 1(1): 38-43, 1998. (in Chinese)

30. X.B. Li, D. Ruan, Y. Xu. A new linguistic-valued aggregation operator to multiple attribute group decision making. Proc. 2007 International Conference on Intelligent Systems and Knowledge Engineering (ISKE2007), Oct 15-16, 2007, Chengdu, China, pp. 846-85 